EGU21-7657, updated on 18 Aug 2021

https://doi.org/10.5194/egusphere-egu21-7657

EGU General Assembly 2021

(c) Author(s) 2021. This work is distributed under

the Creative Commons Attribution 4.0 License.

\title{
Jupiter's X-ray aurora during a mass injection and lo mass loading events observed by XMM-Newton, Hubble, and Hisaki
}

\author{
Affelia Wibisono ${ }^{1,2}$, Graziella Branduardi-Raymont ${ }^{1,2}$, Will Dunn ${ }^{1,2}$, Tomoki Kimura ${ }^{3}$, Andrew \\ Coates $^{1,2}$, Denis Grodent ${ }^{4}$, Zhonghua Yao ${ }^{5}$, Hajime Kita ${ }^{6}$, Pedro Rodriguez ${ }^{7}$, Randy Gladstone ${ }^{8}$, \\ Bertrand Bonfond ${ }^{4}$, and Richard Haythornthwaite ${ }^{1,2}$ \\ ${ }^{1}$ University College London, Mullard Space Science Laboratory, Space and Climate Physics, Dorking, United Kingdom of \\ Great Britain - England, Scotland, Wales (affelia.wibisono.18@ucl.ac.uk) \\ ${ }^{2}$ The Centre for Planetary Science at UCL/Birkbeck, Gower Street, London, UK \\ ${ }^{3}$ Frontier Research Institute for Interdisciplinary Sciences Tohoku University, Japan \\ ${ }^{4}$ Laboratoire de Physique Atmospherique et Planetaire, Universite de Liege, Liege, Belgium \\ ${ }^{5}$ Key Laboratory of Earth and Planetary Physics, Institute of Geology and Geophysics, Chinese Academy of Sciences, Beijing, \\ China \\ ${ }^{6}$ Department of Information and Communication Engineering, Tohoku Institute of Technology, Japan \\ ${ }^{7}$ European Space Astronomy Centre, Madrid, Spain \\ ${ }^{8}$ Southwest Research Institute, San Antonio, Texas, USA
}

Voyager 1 detected the first extra-terrestrial UV auroral emissions when it explored the Jupiter system in 1979 while the planet's X-ray aurora was discovered later that year by the Einstein Observatory. Electrons are accelerated into Jupiter's atmosphere near the poles and excite native molecular and atomic hydrogen. These then release UV photons after returning to the ground state. The same population of precipitating electrons can also emit high energy ( $>2 \mathrm{keV}) \mathrm{X}$-ray photons by bremsstrahlung to produce Jupiter's hard X-ray aurora. At higher latitudes and within the oval of UV and hard X-ray emissions is where the more diffuse UV and low energy ( $<2 \mathrm{keV})$ soft $X$-ray aurorae are found. Charge exchange processes between precipitating ions and neutrals in the gas giant planet's atmosphere are responsible for the soft X-ray emissions.

Simultaneous observations of Jupiter's UV and X-ray aurorae were carried out by the Hubble Space Telescope (HST), Hisaki satellite and XMM-Newton in September 2019 to support Juno's $22^{\text {nd }}$ perijove. Images of the northern far UV aurora by HST showed internally driven dawn storms and injection events occurring at least twice during the observation period. These features are thought to be caused by magnetic reconnection happening in the middle magnetosphere. This subsequently leads to the dipolarization of the field lines which injects hot magnetospheric plasma from the middle to the inner magnetosphere. Hisaki saw an impulsive brightening in the lo plasma torus on the day of the second event showing that there was indeed a large-scale injection that penetrated the central torus in the inner magnetosphere. At this time, the northern aurora brightened in both extreme UV and hard X-ray, which suggests that there was an increase in electron precipitation. There was no response from the soft X-ray aurora, and no quasi-periodic pulsations, often observed in the auroral emissions, were detected during either of the events. $X$ - 
ray spectral analysis reveals that the precipitating ions were iogenic. We conclude that we have witnessed two cases of mass injection in the Jovian inner magnetosphere due to lo mass loading events. 